M. Morales

Nagoya Math. J.

Vol. 191 (2008), 1-19

\title{
SOME NUMERICAL CRITERIA FOR THE NASH PROBLEM ON ARCS FOR SURFACES
}

\author{
MARCEL MORALES
}

\begin{abstract}
Let $(X, O)$ be a germ of a normal surface singularity, $\pi: \tilde{X} \rightarrow X$ be the minimal resolution of singularities and let $A=\left(a_{i, j}\right)$ be the $n \times n$ symmetrical intersection matrix of the exceptional set of $\tilde{X}$. In an old preprint Nash proves that the set of arcs on a surface singularity is a scheme $\mathcal{H}$, and defines a map $\mathcal{N}$ from the set of irreducible components of $\mathcal{H}$ to the set of exceptional components of the minimal resolution of singularities of $(X, O)$. He proved that this map is injective and ask if it is surjective. In this paper we consider the canonical decomposition $\mathcal{H}=\bigcup_{i=1}^{n} \overline{\mathcal{N}}_{i}$ :
\end{abstract}

- For any couple $\left(E_{i}, E_{j}\right)$ of distinct exceptional components, we define Numerical Nash condition $\left(N N_{(i, j)}\right)$. We have that $\left(N N_{(i, j)}\right)$ implies $\overline{\mathcal{N}}_{i} \not \subset \overline{\mathcal{N}}_{j}$. In this paper we prove that $\left(N N_{(i, j)}\right)$ is always true for at least the half of couples $(i, j)$.

- The condition $\left(N N_{(i, j)}\right)$ is true for all couples $(i, j)$ with $i \neq j$, characterizes a certain class of negative definite matrices, that we call Nash matrices. If $A$ is a Nash matrix then the Nash map $\mathcal{N}$ is bijective. In particular our results depend only on $A$ and not on the topological type of the exceptional set.

- We recover and improve considerably almost all results known on this topic and our proofs are new and elementary.

- We give infinitely many other classes of singularities where Nash Conjecture is true.

The proofs are based on my old work [8] and in Plenat [10].

\section{$\S 1$. Introduction}

Let $(X, O)$ be a germ of a normal surface singularity. In an old preprint, published recently by Duke [9], Nash proved that the set of arcs on a surface singularity is a scheme $\mathcal{H}$, and defined a map $\mathcal{N}$ from the set of irreducible components of $\mathcal{H}$ to the set of exceptional components of the minimal res-

Received January 30, 2006.

Revised January 22, 2007.

2000 Mathematics Subject Classification: 14B05, 14E15, 14J17, 32SXX. 
olution of singularities of $(X, O)$. He proved that this map is injective and ask if it is surjective.

Among the principal contributions to this subject we can cite Monique Lejeune-Jalabert [6], [7], Ana Reguera [7], [13], [14], S. Ishii and J. Kollar [5], G. Gonzalez-Sprinberg and Monique Lejeune-Jalabert [4], Camille Plenat [10] and C. Plenat and P. Popescu-Pampu [12]. The study of arcs spaces was further developed by Kontsevich, Denef and Loeser [1] in the theory of motivic integration.

Let $\pi: \tilde{X} \rightarrow X$ be the minimal resolution of singularities, and $E_{1}, \ldots$, $E_{n}$ be the components of the exceptional divisor, Ana Reguera [13] associates to every $E_{i}$ the family of arcs $\mathcal{N}_{i}$ such that the proper transform cuts properly $E_{i}$, the spaces $\overline{\mathcal{N}}_{i}$ are irreducible and give a decomposition of the space of $\operatorname{arcs} \mathcal{H}=\bigcup \overline{\mathcal{N}}_{i}$. In order to give an affirmative answer to the Nash problem it is sufficient to prove that for any $i \neq j$ then $\overline{\mathcal{N}}_{i} \not \subset \overline{\mathcal{N}}_{j}$. In [13] Theorem 1.10, a valuative sufficient condition is given to separate two Nash components, that has been further developed by Camille Plenat [10], Proposition 2.2:

Proposition. ([10]) Let $\pi: \tilde{X} \rightarrow X$ be the minimal resolution of singularities and $E_{1}, \ldots, E_{n}$ be the components of the exceptional divisor, if there exist some $f \in \mathcal{O}_{X, O}$ such that $\operatorname{ord}_{E_{i}}(f)<\operatorname{ord}_{E_{j}}(f)$ then $\overline{\mathcal{N}}_{i} \not \subset \overline{\mathcal{N}}_{j}$.

The following Theorem follows from my work [8] Theorem 1.1 and Lemma 2.2. Remark that in [12] C. Plenat and P. Popescu-Pampu have recently rediscover a similar condition.

TheOrem. ([8]) Let $(X, O)$ be a germ of normal surface singularity, $\pi: \tilde{X} \rightarrow X$ be the minimal resolution of singularities and $E_{1}, \ldots, E_{n}$ be the components of the exceptional divisor. Let $K_{\tilde{X}}$ the canonical divisor on $\tilde{X}$. Let $E$ be an exceptional effective divisor and $Q=\pi_{*} \mathcal{O}_{\tilde{X}}(-E)$,

1. If $-E \cdot E_{i} \geq 2 K \cdot E_{i}$ for all $i=1, \ldots, n$ then $Q \mathcal{O}_{\tilde{X}}=\mathcal{O}_{\tilde{X}}(-E)$.

2. For any general linear combination $f$ of a set of generators of $Q$ we have $\operatorname{div}(f \circ \pi)=\tilde{H}+E$, where $\tilde{H}$ is the proper transform of the cycle defined by $f$.

In Section 2 we will recall some properties on minimal resolutions of singularities, in Section 3 we will introduce the definition of Nash numerical conditions, this is the central point of this work, in Section 4 we will prove 
that Nash numerical conditions depend only on the intersection matrix of the exceptional set and we will establish a connection between the numerical Nash condition and the Gauss's method to squaring a quadratic form. A Nash matrix will be a matrix satisfying the Nash numerical conditions. In Section 5, we characterize some Nash matrices, in Section 6 we consider like star shaped graphs.

In this paper singularities will be considered only through the dual graph of the minimal resolution of singularities and the intersection matrix. The existence of a formal singularity with a given resolution graph (with negative definite intersection matrix) is guaranteed by Grauert [3]. Let us remark that provide equations corresponding to the dual graph of one singularity is in general an open problem.

\section{$\S 2$. On the minimal resolution of singularities}

Remark 1.

- Since $\pi: \tilde{X} \rightarrow X$ is the minimal resolution of singularities of $X$ we have $-K_{\tilde{X}} \cdot E_{i} \leq 0$ for any $i=1, \ldots, n$. This fact will be very important in our proofs. More precisely, for any irreducible component $E_{i}$ of the exceptional divisor, we consider the adjunction formula for (eventually singular) curves

$$
p\left(E_{i}\right)=\frac{E_{i} \cdot\left(E_{i}+K_{\tilde{X}}\right)}{2}+1
$$

where $p\left(E_{i}\right)$ is the genus of $E_{i}$. Recall that $p\left(E_{i}\right) \geq 0$ and $p\left(E_{i}\right)=0$ if and only if $E_{i}$ is a curve of genus zero and self intersection equal to -1 , which is impossible by Castelnuovo theorem since we are assuming that $\pi: \tilde{X} \rightarrow X$ is the minimal resolution of singularities of $X$. As a consequence $K_{\tilde{X}} \cdot E_{i}=2\left(p\left(E_{i}\right)-1\right)-E_{i}^{2} \geq 0$ for any $i=1, \ldots, n$.

- Since the graph of the resolution is connected we have that for any $1 \leq i<k \leq n$ the intersection number $E_{i} \cdot E_{k} \geq 0$ and for each index $k$ there are at least one index $i$ such that $E_{i} \cdot E_{k}>0$.

It follows from the previous item that if $E=\sum_{k=1}^{n} n_{k} E_{k}, n_{k} \in \mathbb{N}$ is an exceptional divisor such that $E \cdot E_{k} \leq-2 K_{\tilde{X}} \cdot E_{k} \leq 0$, for all $k=1, \ldots, n$, then $E$ has full support, i.e. $n_{k}>0$ for all $k=1, \ldots, n$.

- If $E=\sum_{k=1}^{n} n_{k} E_{k}$ with $n_{k} \in \mathbb{N}^{*}$ for $k=1, \ldots, n$, is an exceptional divisor such that $E \cdot E_{k} \leq-2 K_{\tilde{X}} \cdot E_{k}$, then for any $\alpha \in \mathbb{N}^{*}$ we have $(\alpha E) \cdot E_{k} \leq-2 K_{\tilde{X}} \cdot E_{k}$. 
Definition 1. Let $(X, O)$ be a germ of normal surface singularity, $\pi: \tilde{X} \rightarrow X$ be the minimal resolution of singularities, $E_{1}, \ldots, E_{n}$ be the components of the exceptional divisor and $A=\left(a_{i, j}\right)$ with $a_{i, j}=E_{i} \cdot E_{j}$, be the $n \times n$ symmetrical intersection matrix of the exceptional set of $\tilde{X}$. The dual graph $\Gamma$ of the intersection matrix $A$ is defined as follows:

- The vertices of the graph $\Gamma$ are $E_{1}, \ldots, E_{n}$.

- For $i \neq j$ there is an edge between $E_{i}$, and $E_{j}$ if and only if $a_{i, j} \neq 0$.

Remark 2. The graph $\Gamma$ is connected and conversely by a theorem due to Grauert [3], given a $n \times n$ symmetrical negative definite matrix $A=\left(a_{i, j}\right)$ with integer entries, such that $a_{i, i}<0$ and $a_{i, j} \geq 0$ for any $i, j=1, \ldots, n$, $i \neq j$, with a connected graph there exist a singularity with $A$ as intersection matrix.

\section{§3. Numerical Nash condition}

DEFINITION 2. Let $(X, O)$ be a germ of normal surface singularity, $\pi: \tilde{X} \rightarrow X$ be the minimal resolution of singularities and $E_{1}, \ldots, E_{n}$ be the components of the exceptional divisor. Let $K_{\tilde{X}}$ the canonical divisor on $\tilde{X}$. We say that $(X, O)$ satisfies numerical Nash condition for $(i, j)$ if the following condition is fulfilled

$$
\begin{aligned}
& \left(N N_{(i, j)}\right) \quad \exists E=\sum_{k=1}^{n} n_{k} E_{k}, n_{k} \in \mathbb{N}^{*} \\
& \text { with } n_{i}<n_{j} \text { and }-E \cdot E_{k} \geq 2 K_{\tilde{X}} \cdot E_{k}, \forall k=1, \ldots, n .
\end{aligned}
$$

We also say that $(X, O)$ satisfies numerical Nash condition, $(N N)$, if $\left(N N_{(i, j)}\right)$ is true for all couples $(i, j)$, with $i \neq j$.

As an immediate consequence of Proposition of Plenat [10] and my Theorem [8] we have:

COROLlary 1. With the above notations, if $(X, O)$ satisfy numerical Nash condition for $(i, j)$ then $\overline{\mathcal{N}}_{i} \not \subset \overline{\mathcal{N}}_{j}$. In particular if $(N N)$ is true then the Nash problem on arcs has a positive answer.

Proposition 1. With the notations as above. Let $\Gamma$ be the dual graph of the intersection matrix of the exceptional set. If $(N N)$ is true for $\Gamma$, then 
- $(N N)$ is true for any subgraph of $\Gamma$.

- $(N N)$ is true by decreasing the self intersection numbers.

Proof.

- Let us consider a subgraph $G$ of $\Gamma$ and let $I$ be its support. Since $(N N)$ is true for $\Gamma$, for any $i, j \in I, i \neq j$, there exist $E=\sum_{k=1}^{n} n_{k} E_{k}$, $n_{k} \in \mathbb{N}^{*}$ with $n_{i}<n_{j}$ such that $E \cdot E_{k} \leq-2 K_{\tilde{X}} \cdot E_{k}, \forall k=1, \ldots, n$.

It then follows that for any $k \in I$,

$$
\left(\sum_{l \in I} n_{l} E_{l}\right) \cdot E_{k} \leq-2 K_{\tilde{X}} \cdot E_{k}-\sum_{l \notin I} n_{l} E_{l} \cdot E_{k} \leq-2 K_{X^{\prime}} \cdot E_{k}
$$

where $K_{X^{\prime}}$ is the canonical divisor of the minimal resolution singularity $X^{\prime}$, having $G$ as dual graph of the exceptional set. Remark that $K_{X^{\prime}} \cdot E_{k}=K_{\tilde{X}} \cdot E_{k}$.

- In order to prove the second assertion it will be enough to consider one index $k \in\{1, \ldots, n\}$ and the intersection matrix $A^{\prime}=\left(a_{i, j}^{\prime}\right)$ defined by $a_{i, j}^{\prime}=a_{i, j}$ if $(i, j) \neq(k, k)$ and $a_{k, k}^{\prime}=a_{k, k}-1$. Let remark that the matrix $A^{\prime}$ corresponds to a minimal resolution of some isolated singularity, $\pi^{\prime}: \tilde{X}^{\prime} \rightarrow X^{\prime}$, call $E_{1}^{\prime}, \ldots, E_{n}^{\prime}$ the irreducible components of the exceptional set in $\tilde{X}^{\prime}$ and we can assume that $p\left(E_{i}^{\prime}\right)=p\left(E_{i}\right)$. Let $E=\sum_{l=1}^{n} n_{l} E_{l}, n_{l} \in \mathbb{N}^{*}$ with $n_{i}<n_{j}$ such that $E \cdot E_{l} \leq-2 K_{\tilde{X}} \cdot E_{l}$, $\forall l=1, \ldots, n$ and set $E^{\prime}=\sum_{l=1}^{n} n_{l} E_{l}^{\prime}$. By the Remark 1 we can assume that $n_{l} \geq 2$ for any $l=1, \ldots, n$. It follows that

$$
\begin{aligned}
K_{\tilde{X}^{\prime}} \cdot E_{i}^{\prime} & =K_{\tilde{X}} \cdot E_{i} \quad \text { for } i \neq k \\
K_{\tilde{X}^{\prime}} \cdot E_{k}^{\prime} & =K_{\tilde{X}} \cdot E_{k}+1 \\
E^{\prime} \cdot E_{i}^{\prime} & =E \cdot E_{i} \leq-2 K_{\tilde{X}} \cdot E_{i}=-2 K_{\tilde{X}} \cdot E_{i} \quad \text { for } i \neq k \\
E^{\prime} \cdot E_{k}^{\prime} & =E \cdot E_{k}-n_{k} \leq-2 K_{\tilde{X}} \cdot E_{k}-n_{k} \\
& =-2 K_{\tilde{X}^{\prime}} \cdot E_{k}^{\prime}-n_{k}+2 \leq-2 K_{\tilde{X}^{\prime}} \cdot E_{k}^{\prime} .
\end{aligned}
$$

This completes the proof of the second assertion.

\section{§4. Nash matrices, Gauss sequences}

Let $\pi: \tilde{X} \rightarrow X$ be the minimal resolution of singularities and let $A=$ $\left(a_{i, j}\right)$ be the $n \times n$ symmetrical intersection matrix of the exceptional set of $X$, consider an exceptional effective divisor $E=x_{1} E_{1}+\cdots+x_{n} E_{n}$, then

$$
E \cdot E_{k}=x_{1} E_{1} \cdot E_{k}+\cdots+x_{n} E_{n} \cdot E_{k}=x_{1} a_{k, 1}+\cdots+x_{n} a_{k, n} .
$$


Set ${ }^{t} X=\left(x_{1}, \ldots, x_{n}\right),{ }^{t} C=\left(-2 K_{\tilde{X}} \cdot E_{1}, \ldots,-2 K_{\tilde{X}} \cdot E_{n}\right)$ and $c_{i}=-2 K_{\tilde{X}} \cdot E_{i}$, then

1. Corollary 1 can be translated into linear algebra:

If the inequality: $A X \leq C$ has a solution $\left(x_{1}, \ldots, x_{n}\right) \in \mathbb{N}^{n}$ such that $x_{i}<x_{j}$, then $\overline{\mathcal{N}}_{i} \not \subset \overline{\mathcal{N}}_{j}$

2. The condition $\left(N N_{(i, j)}\right)$ is equivalent to the condition:

the inequality: $A X \leq C$ has solutions $\left(x_{1}, \ldots, x_{n}\right) \in \mathbb{N}^{n}$ such that $x_{i}<x_{j}$.

Remark that since $\tilde{X}$ is the minimal resolution we have $-K_{\tilde{X}} \cdot E_{i} \leq 0$ for any $i$. In what follows we allow the intersection matrix $A$ to have rational terms, remark that after multiplication by a convenient integer it will correspond to a singularity.

LEMma 1. Let $(X, O)$ be a germ of a normal surface singularity, $\pi$ : $\tilde{X} \rightarrow X$ be the minimal resolution of singularities. Assume that $\pi$ has only two exceptional components $E_{1}, E_{2}$. Let $A=\left(\begin{array}{cc}-a & c \\ c & -b\end{array}\right)$ the intersection matrix of $E_{1}, E_{2}$. Then

1. $c<a$ if and only if $\left(N N_{(1,2)}\right)$ is true

2. $c<b$ if and only if $\left(N N_{(2,1)}\right)$ is true

3. $c<\min \{a, b\}$ if and only if $(N N)$ is true.

In particular since the quadratic form associated to the matrix $A$ is negative definite, we have $c^{2}<a b$, which implies that either $\overline{\mathcal{N}}_{1} \not \subset \overline{\mathcal{N}}_{2}$ or $\overline{\mathcal{N}}_{2} \not \subset \overline{\mathcal{N}}_{1}$.

Proof. We are looking for solutions $(x, y) \in \mathbb{N}^{*}$ of the system:

$$
\begin{array}{r}
-a x+c y \leq c_{1} \leq 0 \\
c x-b y \leq c_{2} \leq 0
\end{array}
$$

let $D_{1}$ the line of equation $-a x+c y=c_{1}$ and $D_{2}$ the line with equation $c x-b y=c_{2}$, since $A$ is negative definite we have $c^{2}<a b$, which implies $c / b<a / c$, so the relative positions of the lines $D_{1}, D_{2}$, and the set of solutions of the system $(*)$ are represented in figures below. Since these are the unique possible cases we are done. 


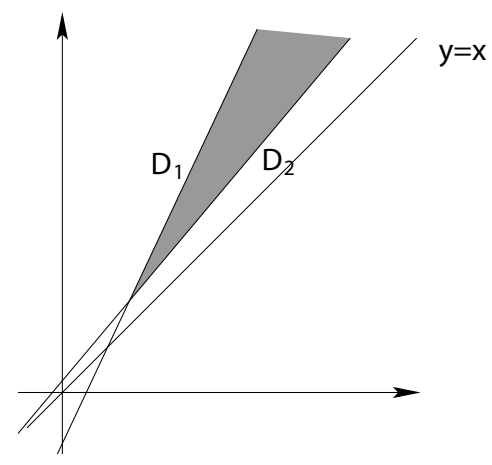

(i) $c<a$ and $b \leq c$

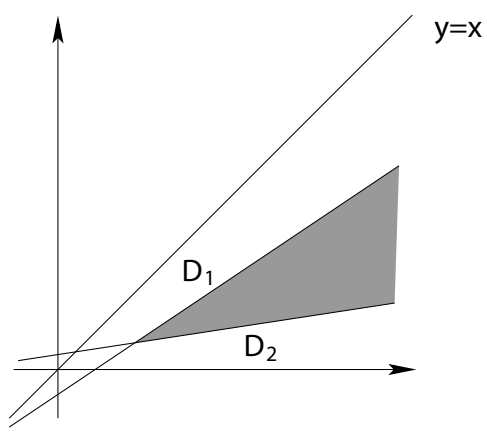

(ii) $c<b$ but $a \leq c$

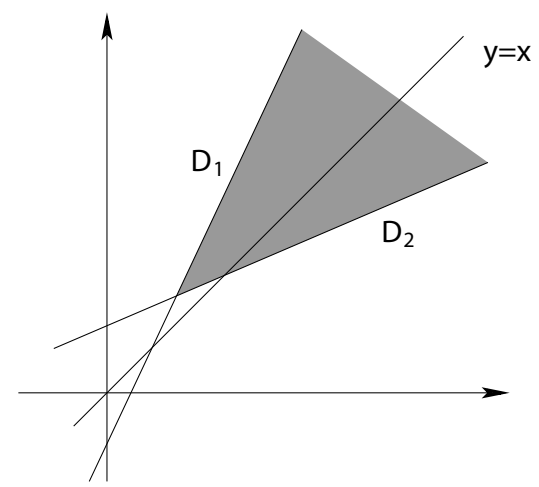

(iii) $c<a$ and $c<b$.

Corollary 2. Let $\pi: \tilde{X} \rightarrow X$ be the minimal resolution of singularities and $E_{1}, \ldots, E_{n}$ be the components of the exceptional divisor, then for any $i \neq j$ either $\overline{\mathcal{N}}_{i} \not \subset \overline{\mathcal{N}}_{j}$ or $\overline{\mathcal{N}}_{j} \not \subset \overline{\mathcal{N}}_{i}$. In any case if $i \neq j$ then $\overline{\mathcal{N}}_{i} \neq \overline{\mathcal{N}}_{j}$. In particular after considering numerical Nash conditions, in order to check if Nash is true, we will be reduced to check at most the half of non inclusion conditions.

We prove the Corollary by induction on $n$. For $n=2$ it was proved in Lemma 1.

Assume $n \geq 3$, by changing the order in the set $E_{1}, \ldots, E_{n}$, we can suppose that $i=1$ and $j=2$, now pick $k$ a positive integer such that $k a_{n, n}<c_{n}$ and put $-a_{n, n} x_{n}=\sum_{1 \leq i \leq n-1} a_{n, i} x_{i}-k a_{n, n}$ in our system $A X \leq C$, then 
we have the inequality: $A^{\prime} X \leq C^{\prime}$ where $a_{i, j}^{\prime}=a_{n, i} a_{n, j}-a_{i, j} a_{n, n}$ for all $i$, $j$ and $c_{j}^{\prime}=\left(-c_{j}+k a_{n, j}\right) a_{n, n}$. By induction hypothesis there exist a vector $S=\left(s_{1}, \ldots, s_{n-1}\right) \in \mathbb{N}^{n}$ solution of the in-equation

$$
A^{\prime} X \leq C^{\prime}
$$

with $s_{1} \neq s_{2}$. Let $s_{n}=a_{n, 1} s_{1}+\cdots+a_{n, n-1} s_{n-1}+k$, then a simple computation shows that the vector $T=\left(-a_{n, n} s_{1}, \ldots,-a_{n, n} s_{n-1}, s_{n}\right)$ is a solution of $A X \leq C$ for $k$ large enough.

Remark that by construction the vector $T$ has strict positive components.

Now we consider the sequences appearing in the proof of the last Corollary.

Definition 3. Let: $a_{i, j}^{(n)}=a_{i, j}$ and for any $2 \leq l \leq n-1$ set $a_{i, j}^{(l)}=$ $a_{i, j}^{(l+1)}-\frac{a_{l+1, i}^{(l+1)} a_{l+1, j}^{(l+1)}}{a_{l+1, l+1}^{(l+1)}}, 1 \leq i, j \leq l$. Also for any $2 \leq l \leq n$ let $C(A)_{i}^{(l)}=$ $\sum_{j=1}^{l} a_{i, j}^{(l)}$. We will also use the notation $C(A)_{i}=C(A)_{i}^{(n)}$.

LEMma 2. The matrices $A^{(l)}=\left(a_{i, j}^{(l)}\right)$ appear naturally when we use the Gauss method to decompose the quadratic form associated to $A$ into a sum of squares. In particular the matrix $A^{(l)}$ are negative definite. For this reason we will call the terms $a_{i, j}^{(l)}$ the Gauss sequence associated to $A$.

Proof. The quadratic form associated to the matrix $A$ is:

$$
Q=\sum_{i=1}^{n} a_{i, i} x_{i}^{2}+2 \sum_{1 \leq i<j \leq n} a_{i, j} x_{i} x_{j}
$$

we follow Gauss method to squaring a quadratic form:

$$
Q=\sum_{i=1}^{n-1} a_{i, i} x_{i}^{2}+2 \sum_{1 \leq i<j \leq n-1} a_{i, j} x_{i} x_{j}+a_{n, n} x_{n}^{2}+2 \sum_{i=1}^{n-1} a_{i, n} x_{i} x_{n}
$$

but

$$
\begin{aligned}
& a_{n, n} x_{n}^{2}+2 \sum_{i=1}^{n-1} a_{i, n} x_{i} x_{n} \\
& \quad=a_{n, n}\left(x_{n}+\sum_{i=1}^{n-1} \frac{a_{i, n}}{a_{n, n}} x_{i}\right)^{2}-\sum_{i=1}^{n-1} \frac{a_{i, n}^{2}}{a_{n, n}} x_{i}^{2}-2 \sum_{1 \leq i<j \leq n-1} \frac{a_{i, n} a_{j, n}}{a_{n, n}} x_{i} x_{j}
\end{aligned}
$$


Hence

$$
\begin{gathered}
Q=a_{n, n}\left(x_{n}+\sum_{i=1}^{n-1} \frac{a_{i, n}}{a_{n, n}} x_{i}\right)^{2}+\sum_{i=1}^{n-1}\left(a_{i, i}-\frac{a_{i, n}^{2}}{a_{n, n}}\right) x_{i}^{2} \\
+2 \sum_{1 \leq i<j \leq n-1}\left(a_{i, j}-\frac{a_{i, n} a_{j, n}}{a_{n, n}}\right) x_{i} x_{j}
\end{gathered}
$$

and $A$ is negative definite if and only if $a_{n, n}<0$ and $A^{(n-1)}$ is negative definite.

Remark 3. 1. By multiplying by a convenient natural number the matrix $A$ has integer coefficients and correspond to some singularities. Our definition does not depend on the topological type of the components of the exceptional divisor.

2. In what follows we will say that for $l \geq 3$ the operation $A^{(l)} \mapsto A^{(l-1)}$ is an algebraic contraction of the exceptional component $E_{l}$ in the graph $\Gamma_{l}$ corresponding to $A^{(l)}$, it is an algebraic operation and this algebraic contraction has no geometry meaning.

We have immediately from Lemma 1 and Corollary 2 that

Proposition 2. Let $\pi: \tilde{X} \rightarrow X$ be the minimal resolution of singularities and let $A=\left(a_{i, j}\right)$ be the $n \times n$ symmetrical intersection matrix of the exceptional set of $X$. Then

1. $a_{1,2}^{(2)}<-a_{1,1}^{(2)}$ if and only if $\left(N N_{(1,2)}\right)$ is true

2. $a_{1,2}^{(2)}<-a_{2,2}^{(2)}$ if and only if $\left(N N_{(2,1)}\right)$ is true

3. $a_{1,2}^{(2)}<\min \left\{-a_{1,1}^{(2)},-a_{2,2}^{(2)}\right\}$ if and only if both $\left(N N_{(1,2)}\right),\left(N N_{(2,1)}\right)$ are true.

TheOREM 1. Let $\pi: \tilde{X} \rightarrow X$ be the minimal resolution of singularities, let $A=\left(a_{i, j}\right)$ be the $n \times n$ symmetrical intersection matrix of the exceptional set of $X$ and let $C(A)_{i}^{(l)}=\sum_{j=1}^{l} a_{i, j}^{(l)}$. For $l \geq 1$, we consider the property:

$\left(*_{l+1}\right) \quad C(A)_{i}^{(l+1)}<0$, for $i=1, \ldots, l+1$.

If $\left(*_{l+1}\right)$ is true for some $l \geq 2$ then $\left(*_{l}\right)$ is true. 
Let $\sigma \in S_{n}$ any permutation of $E_{1}, \ldots, E_{n}$, we denote by $A^{\sigma}$ the corresponding intersection matrix obtained from $A$ by permuting lines and columns. Then $(N N)$ is true if and only if there exist a natural integer $l \geq 1$ such that

$$
\left(*_{l+1}\right) \quad C\left(A^{\sigma}\right)_{i}^{(l+1)}<0, \quad \text { for } i=1, \ldots, l+1, \forall \sigma \in S_{n} .
$$

In particular we recover the following result from [12]: if $C(A)_{i}^{(n)}<0$, for $i=1, \ldots, n$ then the Nash map $\mathcal{N}$ is bijective.

Note that condition $\left(*_{l}\right)$ has a meaning only if $l \geq 2$.

Proof. Assume that $C(A)_{i}^{(l+1)}<0$, for $i=1, \ldots, l+1$, let $i \leq l$, by definition

$$
\begin{gathered}
C(A)_{i}^{(l)}=\sum_{j=1}^{l} a_{i, j}^{(l)}=\sum_{j=1}^{l}\left(a_{i, j}^{(l+1)}-\frac{a_{l+1, i}^{(l+1)} a_{l+1, j}^{(l+1)}}{a_{l+1, l+1}^{(l+1)}}\right) \\
C(A)_{i}^{(l)}=\sum_{j=1}^{l} a_{i, j}^{(l+1)}-\frac{a_{l+1, i}^{(l+1)}}{a_{l+1, l+1}^{(l+1)}} \sum_{j=1}^{l} a_{l+1, j}^{(l+1)}, \\
C(A)_{i}^{(l)}=C(A)_{i}^{(l+1)}-\frac{a_{l+1, i}^{(l+1)}}{a_{l+1, l+1}^{(l+1)}} C(A)_{l+1}^{(l+1)}<0 .
\end{gathered}
$$

The second assertion follows from Proposition 3. Remark that it is not necessary to consider all permutation of $E_{1}, \ldots, E_{n}$.

Definition 4. Let $A=\left(a_{i, j}\right)$ be the $n \times n$ symmetrical negative definite matrix with rational coefficients with $a_{i, i}<0, a_{i, j} \geq 0$ for all $i, j$, $i \neq j$. We say that $A$ is a Nash matrix if for any permutation $\sigma$ of the set $\{1, \ldots, n\} C\left(A^{\sigma}\right)_{1}^{(2)}<0, C\left(A^{\sigma}\right)_{2}^{(2)}<0$.

\section{§5. Trees, cycles, generalized cycles}

We look now for some necessary or sufficient conditions in order to have the condition $(N N)$ true. For the moment we need to recall some notation on graphs.

Definition 5. Let $A=\left(a_{i, j}\right)$ be the $n \times n$ symmetrical negative definite matrix with rational coefficients with $a_{i, i}<0, a_{i, j} \geq 0$ for all $i, j$, 
$i \neq j$. Let $\Gamma$ the dual graph associated to $A$. We say that $E_{i}$ is a leaf of $\Gamma$ if $a_{i, j} \neq 0$ for exactly one index $j \neq i$ i.e. $E_{i}$ is connected to only one other vertex of $\Gamma$. A cycle of $\Gamma$ is a subgraph $\mathcal{C}$ where every vertex is connected to exactly two others vertices of $\mathcal{C}$. A tree is a subgraph with no cycles. Finally a complete subgraph is a subset of $\Gamma$, where every two points are connected.

Lemma 3. Assume that for any point $E_{j}$ of $\Gamma$, we have $C(A)_{j} \leq 0$.

1. For any $l \leq n$ and $j \leq l$ we have $C(A)_{j}^{(l)} \leq 0$

2. If $C(A)_{i}^{(l+1)}<0$ then $C(A)_{i}^{(l)}<0$

3. Let us consider a path $E_{i_{1}}, E_{i_{2}}, \ldots, E_{i_{k}}$ in $\Gamma$, and $C(A)_{i_{k}}<0$. After the algebraic contraction of $E_{i_{k}}, E_{i_{k-1}}, \ldots, E_{i_{3}}$ we will have $C(A)_{i_{2}}^{(2)}<$ 0 .

Proof. The first two assertions follow immediately from the following formula, which is true for any $l \geq 2$, and $1 \leq i \leq l$ :

$$
C(A)_{i}^{(l)}=C(A)_{i}^{(l+1)}-\frac{a_{l+1, i}^{(l+1)}}{a_{l+1, l+1}^{(l+1)}} C(A)_{l+1}^{(l+1)}<0 .
$$

We prove the third assertion by induction on $k$ the length of the path, if $k=2$, there is nothing to do. Now take any $k \geq 3$, then using the above formula we have that $C(A)_{i_{k-1}}^{(n-1)}<0$, by the induction hypothesis we get $C(A)_{i_{2}}^{(n-k+1)}<0$, so by the assertion 1 we are done.

Theorem 2. Let $\pi: \tilde{X} \rightarrow X$ be the minimal resolution of singularities and let $A=\left(a_{i, j}\right)$ be the $n \times n$ symmetrical intersection matrix of the exceptional set of $X$.

1. If $(N N)$ is true then $C(A)_{i}<0$ for any leaf $E_{i}$ of $\Gamma$.

2. Assume that $\Gamma$ is a tree and $C(A)_{i} \leq 0$ for any vertex $E_{i}$ of $\Gamma$. Then $C(A)_{i}<0$ for any leaf $E_{i}$ of $\Gamma$ if and only if $(N N)$ is true.

3. In particular if the above conditions are satisfied the Nash map $\mathcal{N}$ is bijective.

Proof. 
1. Suppose that $(N N)$ is true. Let $E_{i}$ be a leaf of $\Gamma$, we can assume that $i=1$ and $E_{2}$ is the unique vertex connected to $E_{1}$, by the algebraic contraction of all other vertices of $\Gamma$, we will have $a_{1,1}^{(2)}=a_{1,1}$, $a_{1,2}^{(2)}=a_{1,2}$. By Proposition 2 (or Theorem 1) we must have $C(A)_{1}=$ $C(A)_{1}^{(2)}<0$. This concludes the proof of the first statement.

2. The necessary condition was proved before. The proof of the other implication is by induction on $n$. If $n=2$ the hypothesis implies that $(N N)$ is true by Lemma 1 . So assume the case $n-1$ is solved and we prove the case $n$. Take any $i \neq j$. We have two cases

1) Both $E_{i}, E_{j}$ are leaves of $\Gamma$, then $C(A)_{i}^{(n)}<0, C(A)_{j}^{(n)}<0$, by the algebraic contraction of all vertices in $\Gamma$ except $E_{i}, E_{j}$ and applying Lemma 3 , we get that $C(A)_{i}^{(2)}<0, C(A)_{j}^{(2)}<0$, and we are done.

2) At most one of $E_{i}, E_{j}$ is a leaf, then there exist a leaf $E_{k}$, different from $E_{i}, E_{j}$, so after changing the order of the exceptional components we can assume that $i=1, j=2, k=n$, let $E_{l}$ be unique component connected to $E_{n}$. By the algebraic contraction of $E_{n}$, we get the matrix $A^{(n-1)}=\left(a_{i, j}^{(n-1)}\right)$, with $a_{i, j}^{(n-1)}=a_{i, j}$ for any $(i, j) \neq$ $(l, l)$ and $a_{l, l}^{(n-1)}=a_{l, l}-\frac{a_{l, n}^{2}}{a_{n, n}}<0$. It follows that $C(A)_{i}^{(n-1)}=C(A)_{i} \leq$ 0 for $i \neq l$ and $C(A)_{l}^{(n-1)}=C(A)_{l}-\left(\frac{a_{l, n}^{2}}{a_{n, n}}+a_{l, n}\right)<C(A)_{l} \leq 0$. Also the graph corresponding to the matrix $A^{(n-1)}$ is a tree, so by induction hypothesis $\left(N N_{(1,2)}\right)$ and $\left(N N_{(2,1)}\right)$ are true, and we are done.

Remark 4. Inside the class of rational singularities, rational minimal singularities are exactly those for which the graph satisfies the hypothesis of the above theorem. Note that Nash problem's on arcs for (rational) minimal singularities has a positive solution by the work of Ana Reguera [13], also C. Plenat [11] and Fernandez-Sanchez [2] gave different proofs. Our Theorem applies without any restriction on the topological type of the exceptional components and so extends to non rational singularities the mentioned results.

Definition 6. We say that a subgraph $G$ of $\Gamma$ is a generalized cycle if any two vertices of $G$ are connected by a cycle. Remark that a cycle or a complete graph are generalized cycles. 
A generalized cycle is a leaf of $\Gamma$ if at most one vertex of $G$ is connected to one vertex of $\Gamma \backslash G$.

Theorem 3. Let $\pi: \tilde{X} \rightarrow X$ be the minimal resolution of singularities and let $A=\left(a_{i, j}\right)$ be the $n \times n$ symmetrical intersection matrix of the exceptional set of $X$.

1. Assume that the graph $\Gamma$ of the exceptional set is a cycle, with $n \geq 3$, and $C(A)_{i} \leq 0$ for all $i$. Then $(N N)$ is true if and only if $C(A)_{i}<0$ for at least two exceptional components.

2. Suppose that $\Gamma$ is a generalized cycle, $n \geq 3$ and $C(A)_{i} \leq 0$, for any vertex $E_{i}$. Then $(N N)$ is true if and only if $C(A)_{i}<0$, for at least two vertices.

3. In particular if these conditions are fulfilled the Nash map $\mathcal{N}$ is bijective.

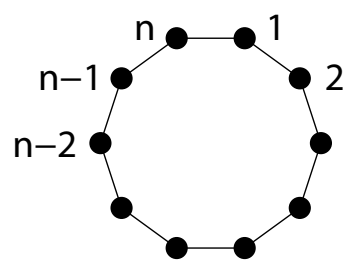

Proof.

1. Assume first that $C(A)_{i}<0$ for at least two exceptional components. We prove that $(N N)$ is true by induction on $n$.

If $n=3$, we make the algebraic contraction of the exceptional fiber $E_{3}$ and we get the matrix:

$$
A^{(2)}=\left(\begin{array}{cc}
a_{1,1}-\frac{a_{1,3}^{2}}{a_{3,3}} & a_{1,2}-\frac{a_{1,3} a_{2,3}}{a_{3,3}} \\
a_{1,2}-\frac{a_{1,3} a_{2,3}}{a_{3,3}} & a_{2,2}-\frac{a_{2,3}^{2}}{a_{3,3}}
\end{array}\right)
$$

It follows that $C(A)_{1}^{(2)}=C(A)_{1}-\left(\frac{a_{1,3}}{a_{3,3}}\left(C(A)_{3}\right)\right)<0$ and $C(A)_{2}^{(2)}=$ $C(A)_{2}-\left(\frac{a_{2,3}}{a_{3,3}}\left(C(A)_{3}\right)\right)<0$ since by hypothesis two over the three numbers $C(A)_{1}, C(A)_{2}, C(A)_{3}$ are strictly negative. So the case $n=3$ is over.

Consider now the case $n \geq 4$. By the algebraic contraction of $E_{n}$, we get the matrix $A^{(n-1)}=\left(a_{i, j}^{(n-1)}\right)$, with $a_{i, j}^{(n-1)}=a_{i, j}$ if $i, j \in$ 
$\{2,3, \ldots, n-2\}$ and $a_{1,1}^{(n-1)}=a_{1,1}-\frac{a_{1, n}^{2}}{a_{n, n}}, a_{1, n-1}^{(n-1)}=-\frac{a_{1, n} a_{n-1, n}}{a_{n, n}}$ and $a_{n-1, n-1}^{(n-1)}=a_{n-1, n-1}-\frac{a_{n-1, n}^{2}}{a_{n, n}}$.

It follows that $C(A)_{i}^{(n-1)}=C(A)_{i} \leq 0$ for $i \in\{2,3, \ldots, n-2\}$, $C(A)_{n-1}^{(n-1)}=C(A)_{n-1}-\left(\frac{a_{n-1, n}}{a_{n, n}}\left(C(A)_{n}\right)\right)$ and $C(A)_{1}^{(n-1)}=C(A)_{1}-$ $\left(\frac{a_{1, n}}{a_{n, n}}\left(C(A)_{n}\right)\right)$. We have to consider three cases:

i) $C(A)_{1}=C(A)_{n-1}=C(A)_{n}=0$ then there are two indexes $i, j \in\{2,3, \ldots, n-2\}$ such that $C(A)_{i}^{(n-1)}<0, C(A)_{j}^{(n-1)}<0$.

ii) $C(A)_{n}<0$, then $C(A)_{n-1}^{(n-1)}, C(A)_{1}^{(n-1)}$ are strictly negative.

iii) at least one of $C(A)_{1}=0, C(A)_{n-1}$ and $C(A)_{n}$ is zero, then either $C(A)_{n-1}^{(n-1)}<0$ or $C(A)_{1}^{(n-1)}<0$.

So the induction hypothesis is verified by $A^{(n-1)}$ and we are done.

Conversely, if $(N N)$ is true and $C(A)_{i}<0$ for at most one index $i$, take any index $j \neq i$, by the algebraic contraction of all other components $E_{k}, k \neq i, j$ we will have $C(A)_{i}^{(2)}=C(A)_{i}=0, C(A)_{j}^{(2)}=C(A)_{j}<0$, this is a contradiction by Proposition 3 .

2. If $\Gamma$ is a generalized cycle, then any two vertices are connected by a cycle and the statement follows from the first item.

EXAMPLE 1. The following matrix and graph correspond to a generalized cycle, for which Nash's problem has an affirmative answer.

$$
A=\left(\begin{array}{cccccccc}
-5 & 1 & 1 & 1 & 1 & 1 & 0 & 0 \\
1 & -6 & 1 & 1 & 0 & 1 & 1 & 0 \\
1 & 1 & -5 & 1 & 0 & 0 & 1 & 1 \\
1 & 1 & 1 & -5 & 1 & 0 & 0 & 1 \\
1 & 0 & 0 & 1 & -2 & 0 & 0 & 0 \\
1 & 1 & 0 & 0 & 0 & -2 & 0 & 0 \\
0 & 1 & 1 & 0 & 0 & 0 & -2 & 0 \\
0 & 0 & 1 & 1 & 0 & 0 & 0 & -3
\end{array}\right)
$$

\section{§6. Like star graphs}

We can improve the above result in the some special situations:

THEOREM 4. Let $\pi: \tilde{X} \rightarrow X$ be the minimal resolution of singularities and let $A=\left(a_{i, j}\right)$ be the $n \times n$ symmetrical intersection matrix of the exceptional set of $X$. Assume that $X$ has a polygon singularity, i.e. the graph of the exceptional set is a star with root $E_{n}$ and all other vertices are leaves. 


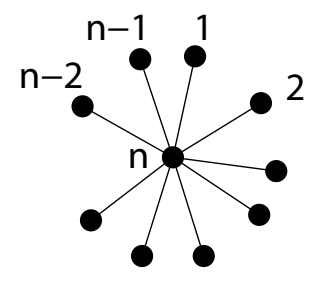

Then $(N N)$ is true if and only if we have the following conditions:

- $\forall i=1, \ldots, n-1 \quad a_{i, i}+a_{i, n}<0$

- $\forall i, j=1, \ldots, n-1 \quad a_{i, i} a_{j, j} \Delta_{n}+a_{j, n}\left(a_{i, i} a_{j, n}-a_{j, j} a_{i, n}\right)<0$

- $\forall i=1, \ldots, n-1 \quad \frac{a_{i, n}}{a_{i, i}}\left(a_{i, i}+a_{i, n}\right)+\Delta_{n}<0$

where $\Delta_{n}=a_{n, n}-\sum_{i=1}^{n-1} \frac{a_{i, n}^{2}}{a_{i, i}}$. We note that $A$ is negative definite if and only if $\Delta_{n}<0$.

Proof. It is enough to compare any two leaves and any leaf with the root. So we consider the following order: $E_{1}, E_{2}, E_{n}, E_{3}, \ldots, E_{n-1}$. After applying the construction above we are reduced to the matrix

$$
A^{(3)}=\left(\begin{array}{ccc}
a_{1,1} & 0 & a_{1, n} \\
0 & a_{2,2} & a_{2, n} \\
a_{1, n} & a_{2, n} & a_{n, n}^{(3)}
\end{array}\right)
$$

where $a_{n, n}^{(3)}=a_{n, n}-\sum_{i=3}^{n-1} \frac{a_{i, n}^{2}}{a_{i, i}}$. We are reduced to consider two cases:

first case: Comparison of $E_{1}, E_{2}$ Again by the construction above we are reduced to the matrix:

$$
A^{(2)}=\left(\begin{array}{cc}
a_{1,1}-\frac{a_{1, n}^{2}}{a_{n, n}^{(3)}} & -\frac{a_{1, n} a_{2, n}}{a_{n, n}^{(3)}} \\
-\frac{a_{1, n} a_{2, n}}{a_{n, n}^{(3)}} & a_{2,2}-\frac{a_{2, n}^{2}}{a_{n, n}^{(3)}}
\end{array}\right)
$$

So $(N N)_{(1,2)}$ and $(N N)_{(2,1)}$ are true if and only if:

$$
\begin{gathered}
a_{1,1}-\frac{a_{1, n}^{2}}{a_{n, n}^{(3)}}-\frac{a_{1, n} a_{2, n}}{a_{n, n}^{(3)}}<0 \\
a_{2,2}-\frac{a_{2, n}^{2}}{a_{n, n}^{(3)}}-\frac{a_{1, n} a_{2, n}}{a_{n, n}^{(3)}}<0
\end{gathered}
$$


by simple computations these are equivalent to:

$$
a_{i, i} a_{j, j} \Delta_{n}+a_{j, n}\left(a_{i, i} a_{j, n}-a_{j, j} a_{i, n}\right)<0
$$

for $\{i, j\}=\{1,2\}$.

Let us consider now the second case: Comparison of $E_{1}, E_{n}$

By the construction above we are reduced to the matrix:

$$
\left(\begin{array}{cc}
a_{1,1} & a_{1, n} \\
a_{1, n} & a_{n, n}^{(3)}-\frac{a_{2, n}^{2}}{a_{2,2}}
\end{array}\right)
$$

So $(N N)_{(1, n)}$ and $(N N)_{(n, 1)}$ are true if and only if: $a_{1,1}+a_{1, n}<0$ and $a_{1, n}+a_{n, n}^{(3)}-\frac{a_{2, n}^{2}}{a_{2,2}}<0$.

After simple computations these are equivalent to: $a_{1,1}+a_{1, n}<0$ and $\frac{a_{1, n}}{a_{1,1}}\left(a_{1,1}+a_{1, n}\right)+\Delta_{n}<0$ Since the choice of the leaves were arbitrary, we are done.

The next corollary follows immediately from the theorem.

Corollary 3. Let $\pi: \tilde{X} \rightarrow X$ be the minimal resolution of singularities and let $A=\left(a_{i, j}\right)$ be the $n \times n$ symmetrical intersection matrix of the exceptional set of $X$. Assume that $X$ has a polygon singularity, the graph of the exceptional set is a star shaped with root $E_{n}$, and $a_{i, n}=1, a_{i, i}=-2$, for $i=1, \ldots, n-1$. Then the matrix $A$ is negative definite if and only if $-a_{n, n}>\frac{n-1}{2}$ and $(N N)$ is true if and only if $-a_{n, n}>\frac{n}{2}$. So if $n$ is odd $(N N)$ is always true, but if $n$ is even it remains open the case $-a_{n, n}=\frac{n}{2}$. By the above theorem only the cases $\left(N N_{(n, i)}\right)$ for $i=1, \ldots, n-1$ are not true.

EXAMPLE 2. Our theorem cannot be applied to the following graph of a sandwich singularity where $a \geq 3$. In fact it follows from the theorem that only $(N N)_{(1,3)}$ is not true. Note that the Nash problem's on arcs for (rational) sandwich singularities has a positive solution by the work of Ana Reguera [14].

$$
A=\left(\begin{array}{cccc}
-a & 0 & 1 & 0 \\
0 & -2 & 1 & 0 \\
1 & 1 & -2 & 1 \\
0 & 0 & 1 & -2
\end{array}\right)
$$

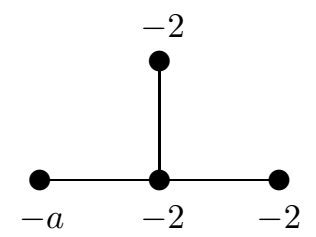


TheOREM 5. Let $\pi: \tilde{X} \rightarrow X$ be the minimal resolution of singularities and let $A=\left(a_{i, j}\right)$ be the $n \times n$ symmetrical intersection matrix of the exceptional set of $X$. Assume that the singularity is like a star, i.e. the graph of the exceptional set is a star with root $E_{0}$ having $s \geq 3$ branches.

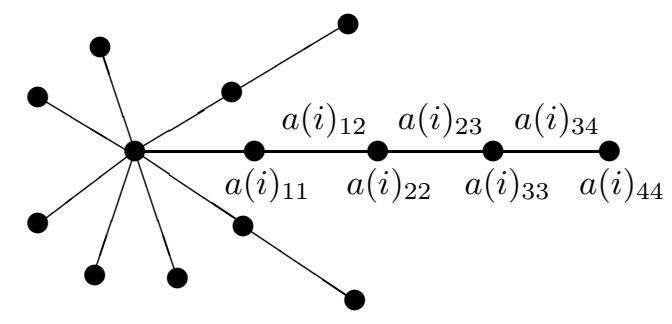

To any branch of the star we associate some continuous fractions expansions $(i=1, \ldots, s ; j \geq 1)$ :

$$
\begin{aligned}
& q_{i}:=a(i)_{1,1}-\frac{a(i)_{1,2}^{2}}{a(i)_{2,2}-\frac{a(i)_{2,3}^{2}}{a(i)_{3,3}-\frac{a(i)_{3,4}^{2}}{\cdots}}} \\
& q_{i, j}:=a(i)_{j, j}-\frac{a(i)_{j, j+1}^{2}}{a(i)_{j+1, j+1}-\frac{a(i)_{j+1, j+2}^{2}}{a(i)_{j+2, j+2}-\frac{a(i)_{j+2, j+3}^{2}}{\cdots}}}
\end{aligned}
$$

Then $(N N)$ is true if we have the following conditions:

- for any leaf $E_{i}$ we have $C(A)_{i}<0$

- for any vertex $E_{i}$ which is not the root $C(A)_{i} \leq 0$

- $\forall i, j=1, \ldots, s, i \neq j, a_{0,0}+a_{0, i}+a_{0, j}-\sum_{k=1, k \neq i, j}^{s} \frac{a_{0, k}^{2}}{q_{k}} \leq 0$

Let $\Delta_{n}=a_{0,0}-\sum_{i=1}^{s} \frac{a_{i, 0}^{2}}{q_{i}}$. We note that $A$ is negative definite if and only if $\Delta_{n}<0$, and $q_{i, j}<0 \forall i=1, \ldots, s ; j \geq 1$.

Proof. Our first step consists to make the algebraic contraction of a whole branch $G_{k}$ of the star $\Gamma$. We reorder the irreducible components of the exceptional set by letting $E_{n}$ to be the leaf of the branch $G_{k}, E_{n-1}$ be the unique vertex connected to $E_{n}, E_{n-2}$ be the unique vertex connected 
to $E_{n-1}$ but distinct from $E_{n}$, and so on until we arrive to the root named always by $E_{0}$, the order in the other branches are arbitrary. We also denote $a_{0, k}:=a(k)_{0,1}$. By the algebraic contraction of $E_{n}$ we will get again a like star graph and a new matrix $A^{(n-1)}=\left(a_{i, j}^{(n-1)}\right)$ given by

$$
a_{i, j}^{(n-1)}=a_{i, j}^{(n)}-\frac{a_{n, i}^{(n)} a_{n, j}^{(n)}}{a_{n, n}^{(n)}},
$$

regarding that our graph is a star we get:

$$
\begin{gathered}
a_{i, j}^{(n-1)}=a_{i, j} \text { if }(i, j) \neq(n-1, n-1) \text { and } \\
a_{n-1, n-1}^{(n-1)}=a_{n-1, n-1}-\frac{a_{n, n-1}^{2}}{a_{n, n}} .
\end{gathered}
$$

Proceeding in this way we can make the algebraic contraction of all the vertices of $G_{k}$, then we will get again a like star graph and a new matrix $A^{\prime}=\left(a_{i, j}^{\prime}\right)$ given by

$$
\begin{gathered}
a_{i, j}^{\prime}=a_{i, j} \quad \text { if }(i, j) \neq(0,0) \text { and } \\
a_{0,0}^{\prime}=a_{0,0}-\frac{a_{0, k}^{2}}{q_{k}} .
\end{gathered}
$$

Now we are ready to prove the claim: we need to compare any two elements in the graph $\Gamma$, these elements are in at most two branches $G_{\alpha}, G_{\beta}$ of the star, we make the algebraic contraction of $s-2$ branches (indexed by a set $I$ ) of the star distinct from $G_{\alpha}, G_{\beta}$ and we get a new graph of type $A_{n}$ and a new matrix $A^{\prime \prime}=\left(a_{i, j}^{\prime \prime}\right)$ given by

$$
\begin{gathered}
a_{i, j}^{\prime \prime}=a_{i, j} \quad \text { if }(i, j) \neq(0,0) \text { and } \\
a_{0,0}^{\prime \prime}=a_{0,0}-\sum_{k \in I} \frac{a_{0, k}^{2}}{q_{k}},
\end{gathered}
$$

the hypothesis of the theorem imply that this special tree satisfies the hypothesis of Theorem 2 and we are done.

Acknowledgment. The author thanks Camille Plenat and the referee for a carefully reading of my paper and helpful comments. 


\section{REFERENCES}

[1] J. Denef and F. Loeser, Germs of arcs on singular varieties and motivic integration, Inv. Math., 135 (1999), 201-232.

[2] J. Fernandez-Sanchez, Equivalence of the Nash conjecture for primitive and sandwiched singularities, Proc. Amer. Math. Soc., 133 (2005), 677-679.

[3] H. Grauert, Uber modifikationen und exceptionnelle analytische Mengen, Math. Annalen, 146 (1962), 331-368.

[4] G. Gonzalez-Sprinberg and M. Lejeune-Jalabert, Families of smooth curves on surface singularities and wedges, Annales Polonici Mathematici, LXVII.2 (1997), 179190.

[5] S. Ishii and J. Kollar, The Nash problem on arc families of singularities, Duke Math. J., 120 (2003), no. 3, 601-620.

[6] M. Lejeune-Jalabert, Courbes tracées sur un germe d'hypersurface, Amer. J. of Math., 112 (1990), 525-568.

[7] M. Lejeune-Jalabert and A. Reguera, Arcs and wedges on sandwiched surfaces singularities, Amer. J. of Math., 121 (1999), 1191-1213.

[8] M. Morales, Clôture intégrale d'idéaux et anneaux gradués Cohen-Macaulay, Géométrie algébrique et applications, La Rabida 1984 (J-M. Aroca, et als, eds.), Hermann, pp. 15-172.

[9] J. F. Nash Jr., Arcs structure of singularities, Duke Math. J., 81 (1995), no. 1, 31-38.

[10] C. Plénat, A Propos du problème des arcs de Nash, Ann. Inst. Fourier., 55 (2005), no. $3,805-823$.

[11] C. Plénat, Résolution du problème des arcs de Nash pour les points doubles rationnels $D_{n}$, Thèse Univ. Paul Sabatier. Toulouse, 2004.

[12] C. Plénat and P. Popescu-Pampu, A class of non-rational surfaces singularities for which the Nash map is bijective, Bulletin Soc. Math. France, to be published.

[13] A. Reguera, Families of Arcs on rational surface singularities, Manuscripta Math., 88 (1995), 321-333.

[14] A. Reguera, A curve selection lemma in spaces of arcs and the image of the Nash map, Compos. Math., 142 (2006), no. 1, 119-130.

Université de Grenoble I, Institut Fourier, UMR 5582, B.P.74

38402 Saint-Martin D'Hères Cedex,

and IUFM de Lyon, 5 rue Anselme

69317 Lyon Cedex

France 